\title{
The effect of adding goal-directed hemodynamic management for elective patients in an established enhanced recovery program for colorectal surgery: results of quasi-experimental pragmatic trial
}

Matthew D. McEvoy ${ }^{1 *}$ DD, Jonathan P. Wanderer ${ }^{1}$, Yaping Shi ${ }^{2}$, Krishnan S. Ramanujan ${ }^{1}$, Timothy M. Geiger ${ }^{3}$, Matthew S. Shotwell ${ }^{2}$, Andrew D. Shaw ${ }^{4}$, Alexander T. Hawkins ${ }^{3}$, Barbara J. Martin ${ }^{5}$, Michael G. Mythen ${ }^{6}$ and Warren S. Sandberg

\begin{abstract}
Background: Recent literature has demonstrated that hemodynamic instability in the intraoperative period places patients at risk of poor outcomes. Furthermore, recent studies have reported that stroke volume optimization and protocolized hemodynamic management may improve perioperative outcomes, especially surgical site infection (SSI), in certain high-risk populations. However, the optimal strategy for intraoperative management of all elective patients within an enhanced recovery program remains to be elucidated.

Methods: We performed a pre-post quasi-experimental study to assess the effect of adding goal-directed hemodynamic therapy to an enhanced recovery program (ERP) for colorectal surgery on SSI and other outcomes. Three groups were compared: "Pre-ERP," defined as historical control (before enhanced recovery program); "ERP," defined as enhanced recovery program using zero fluid balance; and "ERP+GDHT," defined as enhanced recovery program plus goal-directed hemodynamic therapy. Outcomes were obtained through our National Surgical Quality Improvement Program participation.

(Continued on next page)
\end{abstract}

\footnotetext{
* Correspondence: matthew.d.mcevoy@vumc.org

'Department of Anesthesiology, Vanderbilt University Medical Center, 1301 Medical Center Drive, TVC 4648, Nashville, TN 37232, USA
}

Full list of author information is available at the end of the article

(c) The Author(s). 2020 Open Access This article is licensed under a Creative Commons Attribution 4.0 International License, which permits use, sharing, adaptation, distribution and reproduction in any medium or format, as long as you give appropriate credit to the original author(s) and the source, provide a link to the Creative Commons licence, and indicate if changes were made. The images or other third party material in this article are included in the article's Creative Commons licence, unless indicated otherwise in a credit line to the material. If material is not included in the article's Creative Commons licence and your intended use is not permitted by statutory regulation or exceeds the permitted use, you will need to obtain permission directly from the copyright holder. To view a copy of this licence, visit http://creativecommons.org/licenses/by/4.0/ The Creative Commons Public Domain Dedication waiver (http://creativecommons.org/publicdomain/zero/1.0/) applies to the data made available in this article, unless otherwise stated in a credit line to the data. 
(Continued from previous page)

Results: A total of 623 patients were included in the final analysis (Pre-ERP $=246, \mathrm{ERP}=140$, and ERP $+\mathrm{GDHT}=$ 237). Demographics and baseline clinical characteristics were balanced between groups. We did not observe statistically significant differences in SSI or composite complication rates in unadjusted or adjusted analysis. There was no evidence of association between study group and 30-day readmission. American Society of Anesthesiologists status $\geq 3$ and open surgical approach were significantly associated with increased risk of SSI, composite complication, and 30-day readmission ( $p<0.05$ for all) in all groups.

Conclusions: There was no evidence that addition of goal-directed hemodynamic therapy for all patients in an enhanced recovery program for colorectal surgery affects the risk of SSI, composite complications, or 30-day readmission. Further research is needed to investigate whether there is benefit of goal-directed hemodynamic therapy for select high-risk populations.

Trial registration: NCT03189550. Registered 16 June 2017-Retrospectively registered, https://www.clinicaltrials.gov/ ct2/results?cond=\&term=NCT03189550\&cntry=\&state=\&city=\&dist=

Keywords: Enhanced recovery program, Goal-directed, Fluid, Hemodynamics, Colorectal surgery, Monitor, Cardiac output, Blood pressure, Outcomes

\section{Background}

Improving outcomes for surgical patients through goaldirected hemodynamic therapy (GDHT) and enhanced recovery programs (ERPs) has been the focus of major research initiatives for the past 25 years. ERPs have been shown to improve patient outcomes across many types of surgery, with the largest body of evidence surrounding colorectal surgery (Page et al., 2015; Thiele et al., 2015; Zhuang et al., 2015; Wang et al., 2015; Simpson et al., 2015). Of the many components listed for ERPs for colorectal surgery, GDHT and monitoring of cardiac output are given a 'strong' recommendation (Gustafsson et al., 2013; Nygren et al., 2013). However, recent metaanalyses of GDHT trials have yielded mixed results, with one reporting improved outcomes, three reporting a decrease in morbidity but not mortality, and one reporting a decrease in mortality but not morbidity (Feng et al., 2018; Sun et al., 2017; Ripolles-Melchor et al., 2016; Som et al., 2017). Recent trials investigating the effects of goal-directed fluid or hemodynamic therapy showed no benefit (Pestana et al., 2014). On the other hand, three recent large clinical trials have reported positive effects from GDHT for both low to moderate and high-risk surgical patients (Calvo-Vecino et al., 2018; Pearse et al., 2014; Futier et al., 2017).

It is unclear how the results of GDHT trials generalize in the setting of ERPs (Bloomstone \& Dull, 2018). None of the studies included in the meta-analyses of GDHT were in the setting of an ERP (Feng et al., 2018; Sun et al., 2017; Ripolles-Melchor et al., 2016; Som et al., 2017), which is also true for the recent GDHT trials (Calvo-Vecino et al., 2018; Pearse et al., 2014; Futier et al., 2017). Prior research has reported that stroke volume or cardiac output optimization and protocolized intraoperative hemodynamic management may improve outcomes in certain high-risk populations (Cecconi et al., 2013; Arulkumaran et al., 2014). However, the optimal strategy for intraoperative management of patients within an ERP remains to be elucidated (Bloomstone \& Dull, 2018; Gupta \& Gan, 2016). We hypothesized that the addition of continuous, non-invasive cardiac output monitoring with protocolized hemodynamic management for all patients undergoing colorectal surgery within an established ERP would result in decreased rates of surgical site infection (SSI) and improvement in other postoperative outcomes (McEvoy et al., 2016).

\section{Methods}

We performed a pre-post quasi-experimental pragmatic trial where all included patients received the standard of care at the time of their surgical intervention. This study was approved by the Institutional Review Board (IRB) (protocol \#140558) at our institution (Vanderbilt University Medical Center, Nashville, TN, USA), with waiver of requirement for written informed patient consent, as all care components were standard of care. The registration number for the study is NCT03189550 on ClinicalTrials. gov (http://www.clinicaltrials.gov). Matthew D. McEvoy, $\mathrm{MD}$, is the principal investigator; the date of registration is 16 June 2017, which was prior to obtaining data for analysis.

We have previously published the results of our ERP that included the concept of targeting zero fluid balance but did not include algorithm-driven GDHT with continuous non-invasive cardiac output monitoring (McEvoy et al., 2016). Based upon research demonstrating improved outcomes from GDHT with pulse contour analysis, a large-scale quality improvement project was undertaken to advance the ERP for colorectal surgery at our institution through the addition of an intraoperative GDHT management algorithm that included continuous, non-invasive cardiac output monitoring for all 
patients undergoing colorectal surgery. This manuscript adheres to the applicable Standards for Quality Improvement Reporting Excellence (SQUIRE).

Two hundred fifty consecutive patients undergoing elective colorectal surgery including resection of bowel with or without ostomy creation and receiving care within our ERP between 3 March 2015 and 17 February 2017 were included in the study (ERP + GDHT). All care components delivered to these patients were standard of care. A priori, an allocation and analysis plan was created to compare these patients with an historical control group consisting of 250 elective colorectal surgery patients having bowel resection with or without ostomy creation cared for between 9 February 2013 and 26 June 2014, prior to implementation of the colorectal ERP (Pre-ERP), as well as all 146 elective colorectal surgery patients having bowel resection with or without ostomy creation cared for between 27 June 2014 and 2 March 2015, between the inception of the ERP and the start of this quality improvement initiative (ERP). All patients in the groups were included consecutively during their time period. Specifically, the patients in the ERP + GDHT group were enrolled consecutively as noted above. All elective colorectal surgery patients were included from the ERP period. The patients from the pre-ERP group were added to the group by going backwards consecutively from the ERP launch date until 250 patients were included. Emergency cases were excluded, including any case with a preoperative bowel perforation.

Based on prior studies showing a difference in the rate of SSI with GDHT, the primary outcome was the rate of SSI, with secondary outcomes including length of stay, hospital readmission, and a composite complication rate composed of NSQIP (National Surgical Quality Improvement Program) - defined outcomes grouped as respiratory, transfusion, acute kidney injury, urinary tract infection, sepsis, cardiac, and hematologic.

For the patients receiving GDHT, a management algorithm was followed (see Fig. 1). This management protocol was made available to the department as part of the ERP care pathways and was reviewed with all in-room providers (resident or nurse anesthetist) and the assigned anesthesiologist prior to each case. A research assistant was present for monitor calibration and was immediately available for any question concerning the monitor or the protocol. If the anesthesiologist had any questions that involved any clinical decision-making, these were referred to the principal investigator (MDM).

All patient data were collected prospectively through routine clinical care and standardized NSQIP abstracting and stored in the perioperative data warehouse (PDW), an IRB approved data repository (protocol \#120365; waiver of consent approved under that study protocol). Hemodynamic data from the patients in the ERP+GDHT

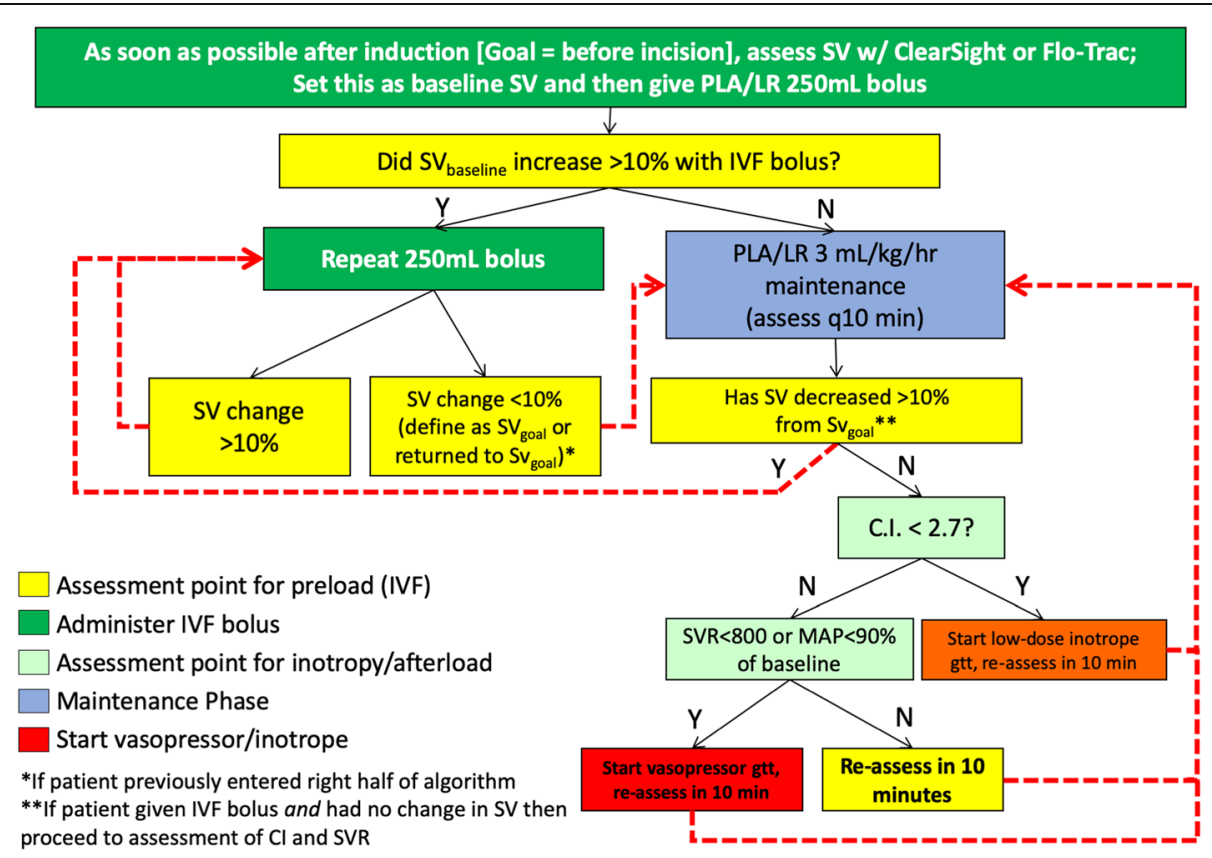

Fig. 1 Goal-directed hemodynamic therapy algorithm. This figure illustrates the goal-directed hemodynamic therapy algorithm that was employed. Stroke volume was monitored with ClearSight sensor and EV1000 clinical platform (Edwards Lifesciences, Irvine, CA). SV: stroke volume; PLA: Plasma-Lyte A; LR: lactated Ringer's; IVF: intravenous fluid; mL: milliliter; kg: kilogram; hr: hour; Cl: cardiac index; SVR: systemic vascular resistance; MAP: mean arterial pressure; gtt: infusion 
group were collected prospectively from the monitoring device (ClearSight sensor and EV1000 clinical platform, Edwards Lifesciences, Irvine, CA, USA) and stored in REDCap after each case (Harris et al., 2009). Patient outcome data were collected by trained abstractors according to the American College of Surgeons (ACS) NSQIP guidelines. Our institution participates in the ACS NSQIP Procedure Targeted program, which includes reporting for all colorectal procedures. Published NSQIP definitions for all the demographic and outcome data were strictly followed (Program ACoSNSQI, n.d.). The abstractors do not deliver clinical care in the operating room and were not aware of the ERP + GDHT implementation until after the study was completed. The Case Mix Index (CMI) of each population is reported as well. CMI is used in USA healthcare to give weight to certain medical conditions around the perioperative period in order to quantify the comorbid status of patient populations. The CMI of a patient population reflects the diversity, clinical complexity, and resource needs of all the patients in that population. A higher CMI indicates a more complex and resourceintensive case load.

\section{Statistical analysis}

Patient demographics and clinical characteristics were summarized using the median (25th and 75th percentile) for continuous variables and percentages for categorical variables. The Kruskal-Wallis test and the Pearson chisquare test were performed as appropriate to compare differences among three study groups (i.e., PreERP, ERP,
ERP + GDHT). Generalized linear mixed effects regression was used to examine the association between study group and the binary outcomes (SSI, readmission in 30 days) while adjusting for a set of pre-specified potential confounders that included age, body mass index (BMI), sex, American Society of Anesthesiologists (ASA) physical status classification, and surgical approach as fixed effects, and surgical procedure (current procedural terminology $[\mathrm{CPT}]$ code) as a random effect. The secular trends over time introduced by unobserved time-dependent confounders were investigated using a segmented regression approach, specifically by adding a time-by-study group interaction in the model. Similarly, linear mixed effects regression was fit for log transformed hospital length of stay (LOS) adjusting for the same set of covariates. Effect estimates were presented using the odds ratio and ratio of medians (OR/RM, 95\% CI, $P$ value).

Finally, in order to evaluate adherence to the GDHT management algorithm, a time-in-target analysis was undertaken to evaluate the time for which the patient had 1,2 , or 3 of the hemodynamic variables in the target range. The hemodynamic algorithm goals evaluated were cardiac index (CI) $>2.7 \mathrm{~L} / \mathrm{min} / \mathrm{m}^{2}$, mean arterial pressure (MAP) $>65$ $\mathrm{mmHg}$, and systemic vascular resistance (SVR) $>800$ dynes"s $/ \mathrm{cm}^{5}$. These variables were based on a compilation of prior studies in GDHT (Feng et al., 2018; Sun et al., 2017).

For a pairwise comparison of study groups, having 250 participants in each group provides approximately $80 \%$ power to detect an odds ratio of 0.45 and 0.20 or smaller with regard to the rate of SSI, assuming that the pre-

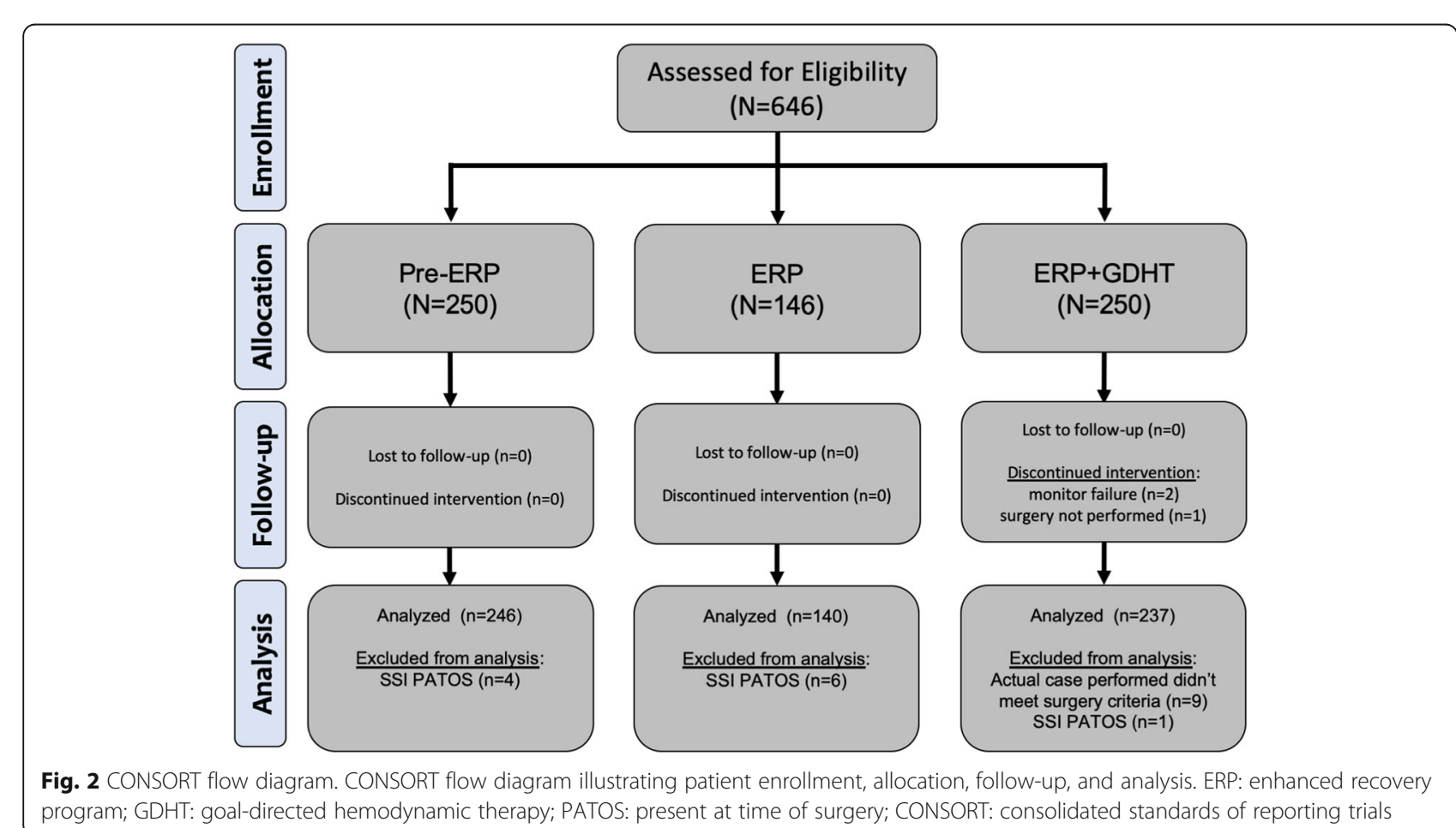


ERP incidence is $18 \%$ and $7 \%$, respectively. Thus, the current study was sufficiently powered to detect a large, clinically meaningful effect of ERP+GDHT on the incidence of SSI in this population. In addition, having 250 participants in each group provides approximately $95 \%$ power to detect a 1-day difference in average LOS, assuming a pre-ERP mean of 5 (SD 3) days.

All analyses were performed using the R Programming Language 3.3.0 (R Foundation for Statistical Computing, Vienna, Austria). We tested for statistical significance at 0.05 significance level.

\section{Results}

A total of 646 patients were assessed for eligibility. Nine patients in the ERP + GDHT group did not meet inclusion criteria based upon the actual surgery performed; two patients were excluded as the non-invasive monitor did not function properly (e.g., routinely displayed error messages or no hemodynamic variables during the case); one patient had the case aborted after induction of anesthesia and before incision owing to the development of unstable atrial fibrillation. After removing 11 patients because of SSI being present at time of surgery (PATOS) (Pre-ERP $=4$, ERP = 6, and ERP + GDHT = 1), the final data set included 623 patients for analysis (Fig. 2).

\section{Patient demographics and case characteristics}

Patient demographics were reasonably balanced between study groups (Table 1). A complete listing of intraoperative variables for all groups can be found in Table 2. Of note, median surgery and anesthesia times were longer and median inspired oxygen and end-tidal carbon dioxide concentrations were higher in the ERP and ERP+ GDHT groups versus Pre-ERP $(P<0.01)$. Total intravenous fluids were lower in the ERP and ERP + GDHT as was use of colloids $(P<0.01)$. Use of vasopressor and inotrope boluses as well as the use of vasopressor infusions increased in the ERP+GDHT periods $(P \leq 0.01)$. However, there was no increase in the use of inotrope infusions.

Surgical case characteristics are shown in Table 3. There were more laparoscopic surgeries in the Pre-ERP group (76.4\%) as compared to ERP (67.9\%) and ERP + GDHT $(65.1 \%)$ groups $(P=0.023)$. There was also a

Table 1 Demographics and baseline characteristics

\begin{tabular}{|c|c|c|c|c|}
\hline Characteristic & $\begin{array}{l}\text { Pre-ERP } \\
(n=246)\end{array}$ & $\begin{array}{l}\text { ERP } \\
(n=140)\end{array}$ & $\begin{array}{l}\text { ERP + GDHT } \\
(n=237)\end{array}$ & $P$ value \\
\hline Age & $58(42,67)$ & $53(39,66)$ & $56(38,66)$ & 0.50 \\
\hline Female [N (\%)] & $132(53.7 \%)$ & $72(51.4 \%)$ & $105(44.5 \%)$ & 0.072 \\
\hline BMI & $26(23,30)$ & $27(24,31)$ & $27(23,32)$ & 0.43 \\
\hline ASA physical status class $[N,(\%)]$ & & & & 0.64 \\
\hline$|/| \mid$ & $110(44.7 \%)$ & $67(47.9 \%)$ & $101(42.8 \%)$ & \\
\hline III/IV & $136(55.3 \%)$ & $73(52.1 \%)$ & $136(57.2 \%)$ & \\
\hline Case mix index & $1.75(1.64,2.57)$ & $2.55(1.66,2.56)$ & $2.55(1.56,2.55)$ & 0.17 \\
\hline Functional health status ${ }^{\mathrm{a}}[N,(\%)]$ & & & & 0.22 \\
\hline Independent & $243(98.8 \%)$ & $139(99.3 \%)$ & $234(98.6 \%)$ & \\
\hline Partially dependent & $3(1.2 \%)$ & $0(0 \%)$ & $3(1.4 \%)$ & \\
\hline Totally dependent & $0(0 \%)$ & $1(0.7 \%)$ & $0(0 \%)$ & \\
\hline \multicolumn{5}{|l|}{ Pre-existing conditions $[N,(\%)]$} \\
\hline Diabetes & $21(8.5 \%)$ & $12(8.6 \%)$ & $21(8.9 \%)$ & 0.98 \\
\hline Current smoker within 1 year & $46(18.7 \%)$ & $27(19.3 \%)$ & $33(14.0 \%)$ & 0.27 \\
\hline Severe COPD & $11(4.5 \%)$ & $2(1.4 \%)$ & $6(2.5 \%)$ & 0.23 \\
\hline Dialysis & $0(0 \%)$ & $2(1.4 \%)$ & $2(0.8 \%)$ & 0.12 \\
\hline Disseminated cancer & $7(2.8 \%)$ & $5(3.6 \%)$ & $3(1.3 \%)$ & 0.35 \\
\hline Open wounds & $4(1.6 \%)$ & $3(2.1 \%)$ & $1(0.4 \%)$ & 0.35 \\
\hline On steroid/immunosuppressant & $76(30.9 \%)$ & $51(36.4 \%)$ & $83(35.2 \%)$ & 0.48 \\
\hline$>10 \%$ weight loss in 6 months & $14(5.7 \%)$ & $10(7.1 \%)$ & $15(6.4 \%)$ & 0.86 \\
\hline Transfusion $72 \mathrm{~h}$ prior to surgery & $0(0 \%)$ & $0(0 \%)$ & $2(0.8 \%)$ & 0.19 \\
\hline
\end{tabular}

${ }^{a}$ Functional health status is only available for 211 patients in the ERP+GDHT cohort. Continuous variables are shown in median (25th, 75 th percentile); categorical data are shown in number and (percentage (\%)).

ERP enhanced recovery program, GDHT goal-directed hemodynamic therapy, $n$ number, \% percentage; $B M I$ body mass index, ASA American Society of Anesthesiologists, COPD chronic obstructive pulmonary disease, SIRS systemic inflammatory response syndrome 
Table 2 Intraoperative variables

\begin{tabular}{|c|c|c|c|c|}
\hline Variable & $\begin{array}{l}\text { Pre-ERP } \\
(n=246)\end{array}$ & $\begin{array}{l}\text { ERP } \\
(n=140)\end{array}$ & $\begin{array}{l}\mathrm{ERP}+\mathrm{GDHT} \\
(n=237)\end{array}$ & $P$ value \\
\hline \multicolumn{5}{|l|}{ Times } \\
\hline Surgery time (min) & $144(108,195)$ & $178(135,237)$ & $165(122,214)$ & $<0.01$ \\
\hline Anesthesia time (min) & $179(144,237)$ & $222(179,282)$ & $206(164,263)$ & $<0.01$ \\
\hline Use of epidural analgesia, $N(\%)$ of patients receiving & $16(6.5 \%)$ & $16(11.4 \%)$ & $11(4.7 \%)$ & 0.04 \\
\hline \multicolumn{5}{|l|}{ Surgical site infection prevention } \\
\hline Pre-incision antibiotics \% & $246(100 \%)$ & $140(100 \%)$ & $236(99.6 \%)$ & 0.44 \\
\hline Median temperature, ${ }^{\circ} \mathrm{C}$ & $36.4(36.1,36.7)$ & $36.3(36.0,36.7)$ & $36.0(35.7,36.3)$ & $<0.01$ \\
\hline Fraction of inspired oxygen & $0.60(0.55,0.80)$ & $0.81(0.76,0.84)$ & $0.75(0.59,0.80)$ & $<0.01$ \\
\hline End-tidal carbon dioxide, $\mathrm{mmHg}$ & $34(32,38)$ & $38(35,39)$ & $37(34,39)$ & $<0.01$ \\
\hline \multicolumn{5}{|l|}{ Glucose } \\
\hline Preoperative, mg/dL & $95(87,106)$ & $102(92,123)$ & $105(92,124)$ & $<0.01$ \\
\hline Recovery room, mg/dL & $146(127,168)$ & $144(122,172)$ & $158(138,178)$ & 0.11 \\
\hline Recovery room glucose $>180, N(\%)$ of patients & $9(3.7 \%)$ & $17(12.1 \%)$ & $17(7.2 \%)$ & 0.01 \\
\hline \multicolumn{5}{|l|}{ Intraoperative intravenous fluid use } \\
\hline Total Intravenous fluids, mL & $2125(1700,2925)$ & $1900(1450,2500)$ & $1800(1500,2300)$ & $<0.01$ \\
\hline Crystalloid, mL & $2000(1600,2800)$ & $1800(1450,2428)$ & $1800(1500,2300)$ & $<0.01$ \\
\hline $\begin{array}{l}\text { Plasmalyte-A or lactated } r \\
\text { inger's, } \mathrm{mL}\end{array}$ & $2000(1500,2600)$ & $1700(1400,2400)$ & $1800(1438,2200)$ & 0.04 \\
\hline Colloid, N (\%) & $36(14.6 \%)$ & $9(6.4 \%)$ & $15(6.4 \%)$ & $<0.01$ \\
\hline Albumin, $N(\%)$ & $35(14.2 \%)$ & $9(6.4 \%)$ & $14(5.9 \%)$ & $<0.01$ \\
\hline Fresh frozen plasma, $N(\%)$ & $0(0 \%)$ & $0(0 \%)$ & $0(0 \%)$ & 0.80 \\
\hline Packed red blood cells, N (\%) & $4(1.6 \%)$ & $0(0 \%)$ & $0(0.8 \%)$ & 0.43 \\
\hline Blood loss, mL & $50(50,100)$ & $75(29,150)$ & $73(30,150)$ & 0.98 \\
\hline \multicolumn{5}{|l|}{ Vasopressor drug use } \\
\hline Vasopressor bolus, N (\%) & $142(57.7 \%)$ & 97 (69.3\%) & $169(71.2 \%)$ & $<0.01$ \\
\hline Vasopressor infusion, $N(\%)$ & $9(3.7 \%)$ & $14(10.0 \%)$ & $53(22.5 \%)$ & $<0.01$ \\
\hline Inotrope bolus, N (\%) & $103(41.9 \%)$ & $50(35.7 \%)$ & $122(51.3 \%)$ & 0.01 \\
\hline Inotrope infusion, N (\%) & $0(0 \%)$ & $1(0.7 \%)$ & $2(0.8 \%)$ & 0.37 \\
\hline \multicolumn{5}{|c|}{ Disposition from operating room (\% going to each destination) } \\
\hline Surgical ward, \% & $246(100 \%)$ & $139(99.3 \%)$ & $236(99.6 \%)$ & 0.46 \\
\hline Intensive care unit, \% & $0(0 \%)$ & $1(0.7 \%)$ & $1(0.4 \%)$ & \\
\hline
\end{tabular}

ERP enhanced recovery program, GDHT goal-directed hemodynamic therapy, median temperature denotes the average of the median temperature of patients in each group, vasopressor phenylephrine, norepinephrine, or vasopressin, intotrope ephedrine, epinephrine, dobutamine, or dopamine. Continuous variables are shown in median (25th, 75th percentile), categorical data are shown in percentage (\%). $P$ values were calculated using the Wilcoxon rank sum test for continuous variables and the Kruskal-Wallis test for categorical variables. $P$ values for any differences among groups

difference in the percentage of proctectomy cases in the Pre-ERP group (12.2\%) as compared to ERP (27.9\%) and ERP + GDHT $(20.8 \%)$ groups $(P<0.001)$.

\section{Outcomes}

There was no evidence of a significant difference in SSI rate or composite complication rate among the three groups before or after adjustment for covariates, although the absolute rates of both outcomes were smaller in ERP + GDHT versus Pre-ERP or ERP samples (Fig. 3a, b). Significantly higher SSI rate was observed for open compared to laparoscopic surgeries overall (15\% vs. $3 \%, P<0.001)$, in Pre-ERP (19\% vs. $4 \%, P<0.001)$, in ERP $(16 \%$ vs. $5 \%, P$ $=0.04)$, and in ERP+GDHT (11\% vs. $1 \%, P<0.001)$. In the adjusted analysis, we examined whether GDHT was associated with reduced SSI rates for either laparoscopic or open surgery patients by testing the interaction of study group by surgery approach. Although the observed rate of SSI was smaller, there was insufficient statistical evidence of a difference for either approach. Due to the rarity of respiratory, transfusion, acute kidney injury, urinary tract infection, sepsis, cardiac, and hematologic complications, as 
Table 3 Case characteristics

\begin{tabular}{llll}
\hline Variable & $\begin{array}{l}\text { Pre-ERP } \\
(\boldsymbol{n}=\mathbf{2 4 6})\end{array}$ & $\begin{array}{l}\text { ERP } \\
(\boldsymbol{n}=\mathbf{1 4 0 )}\end{array}$ & $\begin{array}{l}\text { ERP + GDHT } \\
(\boldsymbol{n}=\mathbf{2 3 7})\end{array}$ \\
\hline $\begin{array}{l}\text { Surgery type } \\
\text { Colectomy }\end{array}$ & $216(87.8 \%)$ & $101(72.1 \%)$ & $188(79.2 \%)$ \\
$\quad$ Proctocolectomy & $30(12.2 \%)$ & $39(27.9 \%)$ & $49(20.8 \%)$ \\
Laparoscopic cases & $188(76.4 \%)$ & $95(67.9 \%)$ & $154(65.1 \%)$ \\
Wound classification & & & \\
$\quad$ Clean & $1(0.4 \%)$ & $0(0 \%)$ & 0.001 \\
$\quad$ Clean contaminated & $157(63.8 \%)$ & $70(50.0 \%)$ & $138(58.1 \%)$ \\
$\quad$ Contaminated & $58(23.6 \%)$ & $46(32.9 \%)$ & $62(26.3 \%)$ \\
$\quad$ Dirty/infected & $30(12.2 \%)$ & $24(17.1 \%)$ & $37(15.7 \%)$ \\
Surgical wound closure & & $139(99.3 \%)$ & 0.085 \\
$\quad$ All layers closed & $244(99.2 \%)$ & & $230(97.0 \%)$ \\
\hline
\end{tabular}

Data are shown as the number and percentage (\%) of patients per group.

ERP enhanced recovery program, GDHT goal-directed hemodynamic therapy, $n$ number

well as readmission within 7 days of surgery, only descriptive statistics are presented (see Additional file 1, Table 1).

The median hospital length of stay (LOS) was 4.2, 3.4, and 4.0 days in Pre-ERP, ERP, and ERP + GDHT groups, respectively $(P=0.10)$. The reduction in LOS from PreERP to ERP was marginal. In unadjusted comparisons, no significant difference in LOS was observed between ERP and Pre-ERP $(P=0.33)$ or between ERP and ERP+ GDHT $(P=0.71)$. However, after adjusting for covariates the median LOS reduced by $12.9 \%$ (95\% CI $0.01-$
23.8, $P=0.04)$ in ERP compared to Pre-ERP. There was no association between ERP + GDHT and readmission in 30 days in the adjusted analysis.

ASA status $\geq 3$ was associated with increased risk of SSI $(P=0.003)$, any complication $(P<0.001)$, and readmission within 30 days $(P=0.027)$. Open (as opposed to laparoscopic) surgical approach was associated with SSI $(P<0.001)$, any complications $(P=0.003)$, and hospital $\operatorname{LOS}(P<0.001)$. There was no significant trend over time for any outcome.

\section{Outcomes by Surgical Approach \\ Pre-ERP $(\mathrm{N}=246) \quad \square \operatorname{ERP}(\mathrm{N}=140) \quad \square \operatorname{ERP}+\mathrm{GDHT}(\mathrm{N}=237)$}

a) Rate of Surgical Site Infection by Surgical Approach

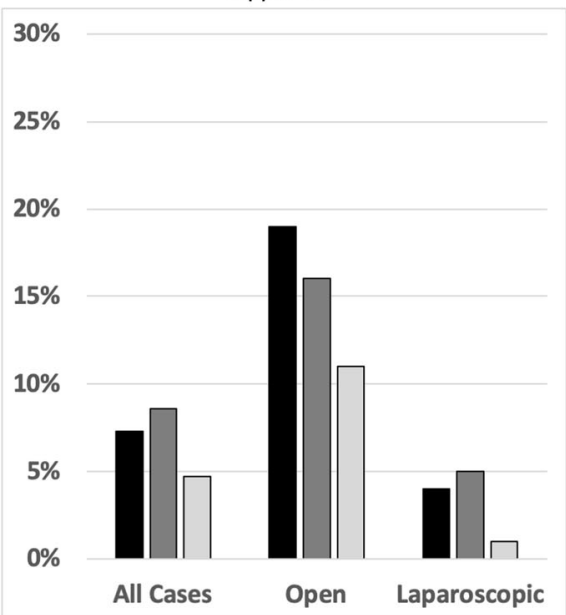

b) Rate of Complications by Surgical Approach (Patients with $\geq 1$ Complication)

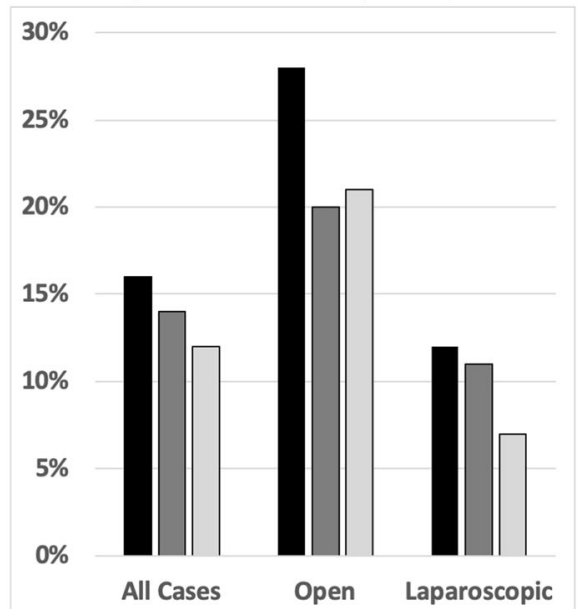

Fig. 3 a, $\mathbf{b}$ Percentage of patients with surgical site infection and any complication by surgical approach. This figure illustrates the percentage of patients with a surgical site infection (a) and overall complications (b) by surgical approach by study phase. There were no statistically significant differences between groups in any phase. ERP: enhanced recovery program; GDHT: goal-directed hemodynamic therapy 


\section{Analysis of goal-directed therapy compliance}

Additional file 1, Table 2 ("Time in Target Analysis") shows the complete results of this analysis. Overall, patients met mean arterial pressure (MAP), systemic vascular resistance (SVR), and cardiac index (CI) goals $94.1 \%, 86.6 \%$, and $85.9 \%$ of the time, respectively. Two out of 3 of the target parameters were maintained $94.7 \%$ of the time. However, all 3 of the parameters were only achieved $36.1 \%$ of the time. There was no association between time-in-target and patient outcomes.

\section{Discussion}

We performed a pragmatic pre-post study to assess the effects of applying continuous cardiac output monitoring and individualized GDHT for all patients undergoing colorectal surgery within an established ERP and found no significant difference in outcomes. There may be several reasons why there was no evidence in this study of a differential benefit for adding GDHT within our ERP. First, SSI (primary outcome) was already at a low rate after the introduction of ERP without GDHT. In fact, our institutional SSI and overall complication rate is within the top performers among NSQIP institutions, which may make further improvement challenging (Hawkins et al., 2019). Additionally, the LOS in patients undergoing colorectal surgery at our institution was lower in all three groups than that reported in any intervention group from the recent large trials in which GDHT was found to be of benefit (Calvo-Vecino et al., 2018; Pearse et al., 2014; Futier et al., 2017). Thus, the ability to show additive benefit of GDHT on complications or LOS for all patients within an established ERP may be more difficult (Bloomstone \& Dull, 2018; Gupta \& Gan, 2016).

It is worthwhile to note that if a GDHT protocol is intended to achieve a high level of compliance with all three hemodynamic targets (i.e., a hemodynamic target zone of normal MAP, afterload, and cardiac output), then the average "dose" in our study is low (i.e., all 3 variables simultaneously in target range $<40 \%$ of time). Our finding of achieving the goal of MAP $>65 \mathrm{mmHg}$ approximately $95 \%$ of the time accords with previous reports (Maheshwari et al., 2018). However, this needs to be further researched, as we found no difference in outcomes even when 2 out of 3 variables were in target at almost all times (95\%) in the ERP + GDHT group. As this was a pragmatic trial that reflects real-world practice, these results should be factored into any subsequent trial design. Perhaps greater computation/automation with closed loop systems administering fluids and medications could achieve a very high level of compliance by not only reacting to changes, but possibly predicting them based on recent hemodynamic patterns (Joosten et al., 2019). Consistent with proposed guidelines (Thiele et al., 2016), our data suggest that GDHT for low risk patients in established ERPs is very unlikely to be cost effective and larger trials are not justified.

However, for higher risk patients, and in particular open surgeries, major complications still occur despite implementation of enhanced recovery principles. In fact, we did find that high-risk patients (e.g., ASA $\geq 3$, open approach) had much higher rates of SSI and all complications than those patients whose procedures were performed laparoscopically. These findings are in line with previous reports and consistent with current guidelines not published when this trial was commended. These patients may be impacted by GDHT, but it should be noted that the incidence of composite complication is impacted by ERPs alone without GDHT. Further research will need to evaluate whether a risk-based algorithm could identify patients who would benefit from GDHT and the associated costs of additional monitoring (Thiele et al., 2016). Accordingly, the design and magnitude of on-going trials would seem justifiable for higher risk patients even in established ERPs (Edwards et al., 2019).

\section{The present study has both strengths and weakness}

A primary weakness is that it was not a prospective, randomized trial, and our data could include sources of bias for which we have not accounted, such as a time bias. We attempted to correct for this, but with an ongoing focus of care standardization and length of stay improvements, we cannot be certain which specific components of care has any causal effect on the outcomes. A strength of this study is the pragmatic, real world implementation of GDHT within an established ERP, as suggested by several consensus guidelines. Yet, this highlights a weakness of our study and possibly of the use of GDHT algorithms in common practice, as we only achieved moderate compliance with the hemodynamic goals. Based on our findings, future trials need to assess whether strict compliance to a GDHT protocol that simultaneously achieves normal preload, afterload, and cardiac output at nearly all timepoints improves outcomes for high-risk patients. If such compliance does improve outcomes, then the means of achieving this level of care in routine practice needs to be determined.

As $80 \%$ of postoperative complications are known to occur in $10-15 \%$ of patients (Sankar et al., 2015), it is important to define the patients for whom employing additional resources and monitoring is of benefit and whether that benefit can still be realized within an established ERP (Bloomstone \& Dull, 2018).

\section{Conclusions}

In a pragmatic pre-post study within an established ERP for colorectal surgery, no benefit was found from implementing GDHT for all patients. Patients with higher co- 
morbidity and those undergoing open surgery are at higher risk of postoperative complications. Future research should be undertaken in larger trials to assess whether GDHT is of benefit for these patients in addition to general principles of enhanced recovery after surgery.

\section{Supplementary Information}

The online version contains supplementary material available at https://doi. org/10.1186/s13741-020-00163-3.

Additional file 1: Table S1. Postoperative American College of Surgeons National Surgical Quality Improvement Program Outcomes. This table lists the postoperative outcomes from the American College of Surgeons National Surgical Quality Improvement Program (ACS NSQIP). Table S2. Time in target analysis. This table shows the complete results of time in target analysis.

\section{Abbreviations}

ACS: American College of Surgeons; ASA: American Society of Anesthesiologists; BMl: Body mass index; Cl: Cardiac index; CONSORT: Consolidated standards of reporting trials; CPT: Current procedural terminology; ERP: Enhanced recovery program; GDHT: Goal-directed hemodynamic therapy; IRB: Institutional review board; LOS: Length of stay; MAP: Mean arterial pressure; NSQIP: National Surgical Quality Improvement Program; OR: Odds ratio; PATOS: Present at time of surgery; PDW: Perioperative data warehouse; RM: Ratio of medians; SSI: Surgical site infection; SVR: Systemic vascular resistance

\section{Acknowledgements}

We would like to thank Martha W. Tanner, BA, editorial assistant, Anesthesiology Department, Vanderbilt University Medical Center, Nashville TN, USA, for her help with preparing the final manuscript.

\section{Authors' contributions}

MDM, JPW, and TMG helped conceive the study design, participated in data collection and analysis with access to the full dataset, and participated in preparing the manuscript. YS, MSS, and ADS participated in data analysis with access to the full dataset and participated in preparing the manuscript KSR and BJM participated in data collection and analysis with access to the full dataset and participated in preparing the manuscript. ATH and MGM participated in review of the data analysis and participated in preparing the manuscript. WSS helped conceive the study design, participated in review of the data analysis with access to the full dataset, and participated in preparing the manuscript. All authors read and approved the final manuscript.

\section{Funding}

This project received funding for monitors and sensors (ClearSight sensor and EV1000 clinical platform), as well as partial support of the research study team from Edwards Lifesciences (Irvine, CA, USA). Edwards Lifesciences approved the investigator-initiated study protocol but did not participate in data collection, data analysis, or preparation of the manuscript.

\section{Availability of data and materials}

The datasets used and/or analyzed during the current study are available from the corresponding author on reasonable request.

\section{Ethics approval and consent to participate}

This study was approved by the Institutional Review Board (IRB) (protoco \#140558) at our institution (Vanderbilt University Medical Center, Nashville, TN, USA), with waiver of requirement for written informed patient consent, as all care components were standard of care. All included patients received the standard of care at the time of their surgical intervention.

\section{Consent for publication}

Not applicable. The study does not contain identifiable details or images from any individual person.

\section{Competing interests}

MDM receives Research funding from Edwards Lifesciences for this project and funding for unrelated research projects from Cheetah Medical and GE Foundation. JPW, YS, KSR, TMG, and MSS have no competing interests to declare. ADS is a consultant for Edwards Lifesciences. ATH and BJM have no competing interests to declare. MGM is University Chair, sponsored by Smiths; director, UCL Discovery Lab; consultant for Edwards Lifesciences; shareholder and scientific advisor, Medical Defense Technologies LLC.; shareholder and director, Clinical Hydration Solutions Itd (patent holder "QUENCH"). WSS is consultant for Edwards Lifesciences.

\section{Author details}

'Department of Anesthesiology, Vanderbilt University Medical Center, 1301 Medical Center Drive, TVC 4648, Nashville, TN 37232, USA. ²Department of Biostatistics, Vanderbilt University Medical Center, 1301 Medical Center Drive, TVC 4648, Nashville, TN 37232, USA. ${ }^{3}$ Department of Surgery, Vanderbilt University Medical Center, 1301 Medical Center Drive, TVC 4648, Nashville, TN 37232, USA. ${ }^{4}$ Department of Anesthesiology \& Pain Medicine, University of Alberta, Edmonton, Alberta, Canada. ${ }^{5}$ Department of Quality, Safety \& Risk Prevention, Vanderbilt University Medical Center, 1301 Medical Center Drive, TVC 4648, Nashville, TN 37232, USA. ' University College London Hospitals National Institute of Health Research Biomedical Research Centre, London, UK.

Received: 30 April 2020 Accepted: 29 October 2020

Published online: 23 November 2020

\section{References}

Arulkumaran N, Corredor C, Hamilton MA, Ball J, Grounds RM, Rhodes A, et al. Cardiac complications associated with goal-directed therapy in high-risk surgical patients: a meta-analysis. Br J Anaesth. 2014;112:648-59.

Bloomstone J, Dull R. Goal-directed fluid therapy in the era of enhanced recovery after surgery: the jury is still out. Comment on Br J Anaesth 2018; 120: 73444. Br J Anaesth 2018;121:673-4.

Calvo-Vecino JM, Ripolles-Melchor J, Mythen MG, Casans-Frances R, Balik A, Artacho JP, et al. Effect of goal-directed haemodynamic therapy on postoperative complications in low-moderate risk surgical patients: a multicentre randomised controlled trial (FEDORA trial). Br J Anaesth. 2018; 120:734-44.

Cecconi M, Corredor C, Arulkumaran N, Abuella G, Ball J, Grounds RM, et al. Clinical review: goal-directed therapy-what is the evidence in surgical patients? The effect on different risk groups. Crit Care. 2013;17:209.

Edwards MR, Forbes G, MacDonald N, Berdunov V, Mihaylova B, Dias P, et al. Optimisation of perioperative cardiovascular management to improve surgical outcome II (OPTIMISE II) trial: study protocol for a multicentre international trial of cardiac output-guided fluid therapy with low-dose inotrope infusion compared with usual care in patients undergoing major elective gastrointestinal surgery. BMJ Open. 2019:9:e023455.

Feng S, Yang S, Xiao W, Wang X, Yang K, Wang T. Effects of perioperative goaldirected fluid therapy combined with the application of alpha-1 adrenergic agonists on postoperative outcomes: a systematic review and meta-analysis. BMC Anesthesiol. 2018;18:113.

Futier E, Lefrant JY, Guinot PG, Godet T, Lorne E, Cuvillon P, et al. Effect of individualized vs standard blood pressure management strategies on postoperative organ dysfunction among high-risk patients undergoing major surgery: a randomized clinical trial. JAMA. 2017;318:1346-57.

Gupta R, Gan TJ. Peri-operative fluid management to enhance recovery. Anaesthesia. 2016;71:40-5.

Gustafsson UO, Scott MJ, Schwenk W, Demartines N, Roulin D, Francis N, McNaught CE, Macfie J, Liberman AS, Soop M, Hill A, Kennedy RH, Lobo DN, Fearon $\mathrm{K}$, Ljungqvist $\mathrm{O}$, Enhanced Recovery After Surgery Society $\mathrm{PPC}$, European Society for Clinical N, Metabolism, International Association for Surgical M, Nutrition. Guidelines for perioperative care in elective colonic surgery: Enhanced Recovery After Surgery (ERAS((R))) Society recommendations. World journal of surgery 2013;37:259-84.

Harris PA, Taylor R, Thielke R, Payne J, Gonzalez N, Conde JG. Research electronic data capture (REDCap)--a metadata-driven methodology and workflow process for providing translational research informatics support. J Biomed Inform. 2009;42:377-81.

Hawkins AT, Geiger TM, King AB, Wanderer JP, Tiwari V Muldoon RL, et al. An enhanced recovery program in colorectal surgery is associated with 
decreased organ level rates of complications: a difference-in-differences analysis. Surg Endosc. 2019:33:2222-30.

Joosten A, Raj Lawrence S, Colesnicenco A, Coeckelenbergh S, Vincent JL, Van der Linden $\mathrm{P}$, et al. Personalized versus protocolized fluid management using noninvasive hemodynamic monitoring (Clearsight system) in patients undergoing moderate-risk abdominal surgery. Anesth Analg. 2019;129:e8e12.

Maheshwari K, Khanna S, Bajracharya GR, Makarova N, Riter Q, Raza S, et al. A randomized trial of continuous noninvasive blood pressure monitoring during noncardiac surgery. Anesth Analg. 2018;127:424-31.

McEvoy MD, Wanderer JP, King AB, Geiger TM, Tiwari V, Terekhov M, et al. A perioperative consult service results in reduction in cost and length of stay for colorectal surgical patients: evidence from a healthcare redesign project. Perioper Med (Lond). 2016;5:3.

Nygren J, Thacker J, Carli F, Fearon KC, Norderval S, Lobo DN, Ljungqvist O, Soop M, Ramirez J, Enhanced Recovery After Surgery Society fPC, European Society for Clinical N, Metabolism, International Association for Surgical M, Nutrition. Guidelines for perioperative care in elective rectal/pelvic surgery: Enhanced Recovery After Surgery (ERAS((R))) Society recommendations. World journal of surgery 2013;37:285-305.

Page AJ, Ejaz A, Spolverato G, Zavadsky T, Grant MC, Galante DJ, et al. Enhanced recovery after surgery protocols for open hepatectomy--physiology, immunomodulation, and implementation. J Gastrointest Surg. 2015;19:387-99.

Pearse RM, Harrison DA, MacDonald N, Gillies MA, Blunt M, Ackland G, et al. Effect of a perioperative, cardiac output-guided hemodynamic therapy algorithm on outcomes following major gastrointestinal surgery: a randomized clinical trial and systematic review. JAMA. 2014;311:2181-90.

Pestana D, Espinosa E, Eden A, Najera D, Collar L, Aldecoa C, et al. Perioperative goal-directed hemodynamic optimization using noninvasive cardiac output monitoring in major abdominal surgery: a prospective, randomized, multicenter, pragmatic trial: POEMAS study (PeriOperative goal-directed thErapy in major abdominal surgery). Anesth Analg. 2014;119:579-87.

Program ACoSNSQI. ACS NSQIP® Semiannual Report Chicago, IL: American College of Surgeons, 2016

Ripolles-Melchor J, Espinosa A, Martinez-Hurtado E, Abad-Gurumeta A, CasansFrances R, Fernandez-Perez C, et al. Perioperative goal-directed hemodynamic therapy in noncardiac surgery: a systematic review and metaanalysis. J Clin Anesth. 2016;28:105-15.

Sankar A, Beattie WS, Wijeysundera DN. How can we identify the high-risk patient? Curr Opin Crit Care. 2015;21:328-35.

Simpson JC, Moonesinghe SR, Grocott MP, Kuper M, McMeeking A, Oliver CM, et al. Enhanced recovery from surgery in the UK: an audit of the enhanced recovery partnership programme 2009-2012. Br J Anaesth. 2015;115:560-8.

Som A, Maitra S, Bhattacharjee S, Baidya DK. Goal directed fluid therapy decreases postoperative morbidity but not mortality in major non-cardiac surgery: a meta-analysis and trial sequential analysis of randomized controlled trials. J Anesth. 2017;31:66-81.

Sun Y, Chai F, Pan C, Romeiser JL, Gan TJ. Effect of perioperative goal-directed hemodynamic therapy on postoperative recovery following major abdominal surgery-a systematic review and meta-analysis of randomized controlled trials. Crit Care. 2017;21:141

Thiele RH, Raghunathan K, Brudney CS, Lobo DN, Martin D, Senagore A, et al. American Society for Enhanced Recovery (ASER) and perioperative quality initiative (POQI) joint consensus statement on perioperative fluid management within an enhanced recovery pathway for colorectal surgery. Perioper Med (Lond). 2016;5:24.

Thiele RH, Rea KM, Turrentine FE, Friel CM, Hassinger TE, McMurry TL, et al. Standardization of care: impact of an enhanced recovery protocol on length of stay, complications, and direct costs after colorectal surgery. J Am Coll Surg. 2015;220:430-43.

Wang C, Chen HN, Zhou ZG. Two-day hospital stay after laparoscopic colorectal surgery: is enhanced recovery program a healthcare system-specific issue? World J Surg. 2015;39:1329-30

Zhuang $\mathrm{CL}$, Huang DD, Chen FF, Zhou CJ, Zheng BS, Chen BC, et al. Laparoscopic versus open colorectal surgery within enhanced recovery after surgery programs: a systematic review and meta-analysis of randomized controlled trials. Surg Endosc. 2015;29:2091-100.

\section{Publisher's Note}

Springer Nature remains neutral with regard to jurisdictional claims in published maps and institutional affiliations.

Ready to submit your research? Choose BMC and benefit from:

- fast, convenient online submission

- thorough peer review by experienced researchers in your field

- rapid publication on acceptance

- support for research data, including large and complex data types

- gold Open Access which fosters wider collaboration and increased citations

- maximum visibility for your research: over $100 \mathrm{M}$ website views per year

At BMC, research is always in progress.

Learn more biomedcentral.com/submissions 